\title{
Effectiveness of a Mindfulness-Based Childbirth Education pilot study on maternal
}

self-efficacy and fear of childbirth

Précis

Meditation and skills based education may enhance self efficacy and reduce fear in nulliparous women with low risk, singleton pregnancies 


\section{ABSTRACT}

Introduction: This pilot study tested the feasibility and effectiveness of using MindfulnessBased Childbirth Education (MBCE), a novel integration of mindfulness meditation and skills-based childbirth education, for mental health promotion with pregnant women. The MBCE protocol aimed to reduce fear of birth, anxiety and stress and improve maternal self efficacy. This pilot study also aimed to determine the acceptability and feasibility of the MBCE protocol.

Methods: A single arm pilot study of the MBCE intervention using a repeated measures design was used to analyze data before and after the MBCE intervention to determine change trends with key outcome variables: mindfulness; depression, anxiety and stress; childbirth self-efficacy; and fear of childbirth. Pregnant women (18-28 weeks gestation) and their support companions attended weekly MBCE group sessions over eight weeks in an Australian community setting.

Results: Of the 18 women who began and completed the intervention, missing data allowed for complete data from 12 participants to be analysed. Statistically significant improvements and large effect sizes were observed for childbirth self-efficacy and fear of childbirth. Improvements in depression, mindfulness and birth outcome expectations approached significance but were underpowered. At post-natal follow-up significant improvements were found in anxiety, while improvements in mindfulness, stress and fear of birth were significant at a less conservative alpha level.

Discussion: This pilot study demonstrated that a blended mindfulness and skills based childbirth education intervention was acceptable to women and is associated with improvements in women's sense of control and confidence in giving birth. Previous findings that low self-efficacy and high childbirth fear are linked to greater labor pain, stress reactivity, and trauma, suggest the observed improvements in these variables have important implications for improving maternal mental health and associated child health outcomes. Ways in which these outcomes can be achieved through improved childbirth education are discussed. 


\section{Quick Points}

- Mindfulness-Based Childbirth Education (MBCE) is a novel integration of mindfulness meditation and skills-based childbirth education focusing on mindfulness, communication and decision making skills, to target self-efficacy and reduce fear and stress.

- The results of this pilot study demonstrate that Mindfulness Based Childbirth Education is associated with feelings of empowerment and confidence in women, as evidenced by improvements in childbirth fear and self-efficacy after the program and improvements in anxiety after giving birth.

- These findings provide justification for pursuing further research into MBCE as a childbirth education program which targets mental health and self efficacy. 


\section{INTRODUCTION}

Research suggests that traditional childbirth education does not actively target mental health improvement (such as stress reduction), either of expectant mothers or their birth support partner. ${ }^{1}$ This is concerning due to the relationship between prenatal stress and both postnatal maternal outcomes and fetal development. Maternal stress is associated with shorter gestation periods, low birth weight and is a risk factor for delay in mental and motor development in infants. ${ }^{2}$ Longitudinal studies have also highlighted the impact of maternal stress during pregnancy on subsequent negative childhood behavior. ${ }^{3}$

Mindfulness-based interventions are recognized for their potential in reducing stress and improving psychological functioning. ${ }^{4}$ Mindfulness involves the cultivation of moment-tomoment awareness of experience with a non-judgmental attitude. Substantial evidence has attested to the benefits of mindfulness-based interventions in treating and preventing psychological dysfunction in a broad spectrum of people, ${ }^{4}$ suggesting that cultivating mindfulness may have mental health benefits for pregnant women and their birth support partner. One recent pilot study of a mindfulness-based prenatal intervention demonstrated significant increases in positive affect, along with decreases in anxiety, depression and negative affect. ${ }^{5}$

The present study sought to build on this research by developing a prenatal group intervention that targeted maternal mental health using mindfulness meditation and an empowerment model of education. ${ }^{6}$ The aim of this latter aspect of the blended approach was to improve mental health by improving self-efficacy and reducing fear of birth, given that these factors have been linked to improved birth outcomes. For example, improving the self-efficacy of pregnant and birthing women can help to reduce perceived pain during childbirth $^{7}$ and act as a preventative measure for women who may experience psychological trauma of childbirth. ${ }^{8}$ Additionally, improved self-efficacy is associated with lower psychological and physiological stress reactivity, which is important given the links between maternal stress and child development. 3,9

The resulting protocol, Mindfulness Based Childbirth Education (MBCE), integrates a skills- 
based prenatal education program with Mindfulness Based Stress Reduction (MBSR), a widely validated group meditation intervention. An active skills-based approach to childbirth education, focusing on communication and decision making skills, was used to target self-efficacy and reduce fear, as evidence suggests such approaches might enhance women's ability to feel part of the labor experience ${ }^{10}$ whilst equipping women with essential information and skills for early parenting. ${ }^{11}$

The present pilot study tested the outcomes and acceptability of the MBCE protocol for first time mothers and their birth support partners in order to determine whether further investigation using a larger controlled trial is warranted. We hypothesized that mindfulness meditation combined with an educational empowerment model would be acceptable to participants and would be associated with increased self-efficacy and mindfulness, along with reduced childbirth fear, stress, depression and anxiety.

\section{METHODS}

\section{Design}

This single arm pilot study used a pre-experimental repeated measures design to collect and analyze data before and after the MBCE intervention, in order to determine change trends in mindfulness; depression, anxiety, stress; childbirth self-efficacy and fear. Effect sizes and post-hoc power were calculated to inform a future clinical trial.

\section{Inclusion and Exclusion Criteria}

To be included, pregnant women (between 18 and 28 weeks gestation) had to be healthy, nulliparous women with singleton pregnancies, English speaking and aged over 18 years. Exclusion criteria included: 1) women taking medication for diagnosed mental illness (eg, anti-depressants, anti-anxiety or anti-psychotic medication); 2) women with complicated or high-risk pregnancies; and 3) women taking illicit drugs. Birth support partners were invited to attend; however, inclusion or exclusion criteria did not apply to birth support partners. Having a support person was not a requirement of participation.

\section{Recruitment}


Participants were recruited through the community. Contact was made via flyers at hospitals, birth centres and organizations that offer prenatal education, and through newspaper articles, online pregnancy forums and email lists. Interested participants self referred.

\section{Procedure}

This study was approved by The University of Western Australia Human Research Ethics Committee (HREC). Participants registered their interest via email and those who met the inclusion criteria were invited to commence the course. Participants completed the pre MBCE questionnaire package and received books and compact discs (CDs) with mindfulness homework practice prior to beginning session one. At the eighth week of the course, following the final class, participants completed the post MBCE questionnaire package Approximately 3-12 weeks following the birth of their baby, participants were contacted to obtain data on birth outcomes and complete a modified questionnaire package with instruments to measure depression, anxiety, stress, mindful awareness and childbirth fear. Qualitative data were also gathered at this point using focus groups.

\section{MBCE Program}

The MBCE protocol was developed specifically for this study and included participants as active learners through group work, role plays and decision making practice using the BRAIN (Benefits, Risks, Alternatives, Intuition, Nothing) model ${ }^{12}$ for decision making, as well as incorporating daily mindfulness meditation homework. The program ran over eight consecutive weeks; each session was approximately 2.5 hours. Pregnant women and their birth support partner (husband, partner, mother, or friend) attended. Participants had homework CDs with mindfulness meditation instructions and a workbook to use during the week between sessions. Daily practice of techniques learned was encouraged. Additionally, participants had assigned reading, usually related to prenatal information content. Each session was run by the principal investigator (JB), a qualified childbirth educator, specialist antenatal yoga teacher, and mindfulness meditation teacher. Sessions were co-facilitated by an assistant who was a registered yoga and meditation teacher. 
Prenatal education and mindfulness was incorporated into each session. The education part of the program provided women and their support person with knowledge and skills to assist in making informed choices regarding their pregnancy, birth and parenting. In order to meet this aim, participants were provided with evidence-based information regarding their choices. Participants engaged in a wide range of learning activities (group discussion, role plays, problem solving activities) to prepare for birth and early parenting, and were taught mindfulness exercises/meditations to develop non-reactive, present moment awareness. They learned how to apply the practice of mindfulness to discomfort during pregnancy, labor pain, and early parenting.

\section{Questionnaire Package}

The following well-validated self-report measures were administered before and after the intervention (reliability in parentheses): The Mindful Attention Awareness Scale (MAAS) ${ }^{13}$ measured mindfulness (Cronbach's $\alpha$.87); the Depression Anxiety Stress Scales (DASS-21) ${ }^{14}$ measured depression, anxiety and stress (Cronbach's $\alpha .87$ to .94); the Edinburgh Post Natal Depression Scale (EPDS) ${ }^{15}$ measured prenatal depression (Cronbach's $\alpha .87$ ); the Childbirth Self-Efficacy Inventory (CBSEI) ${ }^{16}$ measured self efficacy and birth outcome expectancies (Cronbach's $\alpha .86$ to .96); and the Wijma Delivery Expectancy Questionnaire (W-DEQ) ${ }^{17}$ measured fear of childbirth before labor (version A) and afterwards (version B). Split-half reliability for the W-DEQ was .87 or greater. The variables measured at postnatal follow-up were mindfulness (MAAS), childbirth fear (W-DEQ), and depression, anxiety and stress (DASS-21).

\section{Data Screening and Analysis}

Data were entered into the PASW Statistics 18.0 for Mac software (SPSS Inc., 2010, Cary NC, USA) for screening and analysis. Using the Shapiro-Wilk test and visual inspection of histograms, it was determined that the variables met assumptions of normality required for paired samples $t$ tests. An outlier was deleted on the postnatal depression (EPDS) variable due to its skewing effect on the small sample. Due to a large number of missing values, prepost change data were only analysed for 12 of the 18 women who completed the course. Not all participants provided complete follow-up assessments, resulting in a different 
sample size for the follow-up analyses. Effect sizes for pre-post MBCE changes were calculated using Cohen's $d$ statistic. Being a within-subjects study, a correction was applied for the dependence among means, using Morris and DeShon's equation 8.18

\section{RESULTS}

\section{Sample Characteristics}

Demographic characteristics of pregnant women participating in MBCE, as well as characteristics of their subsequent births, are presented in Table 1.

\section{Variable Changes Over Eight Week MBCE Program}

Pre- and post-MBCE intervention scores for each variable are summarised in Table 2. Participants had significantly more self-efficacy, more positive expectations of their births, and were less fearful of giving birth after completing the program. These results were most robust for self-efficacy and fear of birth, which were significant at the Bonferroni-corrected alpha level of $P=.006$. Mindfulness and depression, as measured by the DASS-21, trended toward improvement after the program, but results were not statistically significant.

Pre-intervention scores were compared with follow-up scores on measures of mindfulness (MAAS), fear of birth (W-DEQ), depression, anxiety and stress (DASS). Significant improvements were observed for all these variables, except depression (Table 3).

\section{Effect Size and Power Analyses}

Table 4 summarises the effect sizes for pre- to post-intervention changes and the power obtained (post-hoc) in each test, as well as the sample size required to adequately power (at .80) another study using the obtained effect sizes for each variable of this study. Large effects were found for self-efficacy and fear of birth, the only two variables that were adequately powered. Outcome expectations approached a large effect size and adequate power. Medium effect sizes were found in several variables (mindfulness, depression, and anxiety), although these were underpowered.

\section{Adherence and Acceptability}


Compliance with the intervention was high, with all 18 enrolling participants completing the program. However, adherence to the daily meditation homework was fairly low. The average number of meditation practice sessions per week ranged from 1.8 to 5.8 , with a mean of 3.6 (1.1) practice sessions per week over the 8-week intervention. The average total number of minutes spent meditating at home was 102.8 (49.2), ranging from 30.0 to 191.2 over the whole intervention.

Qualitative data gathered from focus groups conducted at post-birth follow-up suggested participants benefited from the intervention. This data is presented in more detail separately. ${ }^{19}$ Participants reported feeling a sense of community and empowerment from the course. They reported that the MBCE program helped them to be active participants and decision makers in their pregnancy and labor. ${ }^{19}$ These women said that the experiential, active learning components of the course were very engaging. Mindfulness exercises were described as enjoyable during sessions, and the mindfulness skills were reported to be particularly beneficial following birth. However, some participants found the daily mindfulness practice onerous. Some women also suggested that the time of the course be changed to morning rather than lunch time, and that the class duration be shortened

\section{DISCUSSION}

This pilot study tested the feasibility of using Mindfulness-Based Childbirth Education (MBCE), a novel integration of mindfulness meditation and skills-based childbirth education, for mental health promotion with pregnant women. The study involved developing, refining and delivering the MBCE protocol to a small group of pregnant women and their birth support partners. The perfect retention rate and generally positive comments provided in focus groups after birth provide evidence of feasibility and acceptability of the MBCE intervention, as predicted. It was also hypothesised that by the end of the intervention, pregnant women would demonstrate a range of improvements in mental health outcomes. This prediction was largely supported, with the largest effects being observed for childbirth self-efficacy and fear of childbirth, suggesting that women felt more confident and less fearful about birthing a baby after completing MBCE. Results also showed smaller effects for depression and mindfulness immediately following the 
intervention. Interestingly, results at follow-up were more favourable than at post-test. This is supportive of findings from a qualitative arm of the of MBCE study, where participants reported that the benefits of the intervention extended into the postnatal period, particularly those associated with mindfulness and peer support. ${ }^{19}$

Given the lack of a control group and the small sample used in the present study, it is impossible to attribute the observed outcomes to the MBCE intervention. However, the very large effect sizes associated with the improvements in self-efficacy and childbirth fear suggest that these effects relate in some way to the intervention rather than to chance effects or natural processes such as regression to the mean. While detailed interpretation of the $t$-tests from this small data set is not warranted, it is noteworthy that self-efficacy and childbirth fear showed the largest effects out of all the mental health variables measured. This result suggests these variables may be of most relevance to future controlled studies of MBCE and warrant investigation in terms of the understanding the active ingredients in MBCE.

The mindfulness practice appeared to form a less central component of the intervention than originally intended. While all participants engaged in the home mindfulness homework to some extent, none did so daily as prescribed. This suggests that future trials of MBCE could emphasize home practice and explore barriers to this. Participants may have found home practice too onerous. A theme that emerged in a qualitative study of MBCE was that the mindfulness practice was most useful during labor and early parenting, ${ }^{19}$ which might explain the limited adherence in pregnancy. In light of this, the empowerment education component of MBCE was a central feature of the intervention and should continue to be explored in future research.

The results of this pilot study provide justification for pursuing further research into MBCE. A well powered randomized controlled trial comparing MBCE against treatment as usual or a waitlist control would be the next step in evaluating the intervention. The large effect sizes observed for self-efficacy and childbirth fear suggest that 15 participants in each condition would adequately power a study focused on similar variables. However, other 
mental health outcomes such as depression, anxiety and stress are also theoretically relevant and showed modest improvement; a sample that can adequately detect a medium effect size at a power of .80 would be desirable, suggesting 51 participants in each group for such a trial.

Future studies could also explore the mechanisms of change since one limitation of the current study design was that there were several possible active ingredients (eg, mindfulness, skills-based education, group support, non-specific factors). Although not a limitation clinically, from a research perspective it makes testing and refining the protocol challenging. Once the efficacy of the MBCE program is tested in a larger controlled trial, further studies might use component analysis and active control groups to examine active ingredients (eg, comparing MBCE to the same intervention without the mindfulness component).

Notwithstanding these limitations and suggestions for further research, the present study delivered several important findings. It showed that a blended mindfulness and skills-based prenatal education intervention was both acceptable and feasible. The Mindfulness Based Childbirth Education intervention was associated with feelings of empowerment and confidence in women, as evidenced by improvements in childbirth fear and self-efficacy after the program and improvements in anxiety after giving birth. Given the ubiquity of prenatal education in western countries but a lack of commensurate psychological benefits for pregnant women, these findings offer promising evidence to improve the impact of prenatal education on maternal mental health outcomes. 
Table 1. Demographic and Labor Characteristics of Pregnant Women Participating in MBCE Intervention $(\mathrm{N}=18)$

Characteristic

Values

Age, mean (SD), y

Marital status, n (\%)

Married/De facto

$16(89)$

Single

Education, $\mathbf{n}(\%)$

Undergraduate degree

$10(56)$

Postgraduate degree

Planned model of antenatal care, $n$ (\%)

Obstetrician

Midwife

Shared (midwife/general practitioner)

Birthing facility, n (\%)

Private hospital

$10(56)$

Public hospital/birth centre

Commencement of labour, n (\%)

Spontaneous

$10(56)$

Induced

$8(44)$ 
Type of birth, $\mathbf{n}(\%)$

Caesarian

$11(61)$

Vaginal

7 (39)

Established breastfeeding after birth, n (\%)

$18(100)$ 
Table 2. Significance of Changes in Outcome Measures Between Pre- and Post-MBCE Intervention for Pregnant Women $(\mathrm{N}=12)$

\begin{tabular}{lccc} 
Variable (Measure) & $\begin{array}{l}\text { Pre-MBCE } \\
\text { Mean (SD) }\end{array}$ & $\begin{array}{l}\text { Post-MBCE } \\
\text { Mean (SD) }\end{array}$ & P value $^{\mathrm{a}}$ \\
\hline Self Efficacy (CBSEI) & $171.69(42.10)$ & $224.54(25.88)$ & $.001^{\mathrm{b}}$ \\
Outcome Expectations & $236.92(37.21)$ & $254.25(24.19)$ & $.020^{\mathrm{a}}$ \\
(CBSEI) & $7.33(5.07)$ & $7.00(2.83)$ & .058 \\
Postnatal Depression & & & \\
(EPNDS) & $4.04(.84)$ & $4.47(.51)$ & $<.001^{\mathrm{b}}$ \\
Mindfulness (MAAS) & $61.42(13.62)$ & $38.92(14.84)$ & .070 \\
Fear of Birth (W-DEQ) & $5.83(5.29)$ & $3.17(3.46)$ & .605 \\
Depression (DASS-21) & $8.33(7.57)$ & $6.00(7.53)$ & .255 \\
Anxiety (DASS-21) & $9.83(5.42)$ & $11.50(6.45)$ & \\
Stress (DASS-21) & & & \\
\hline Ab & & & \\
\hline
\end{tabular}

Abbreviations: MBCE, Mindfulness-Based Childbirth Education; CBSEI, Childbirth SelfEfficacy Inventory; EPNDS, Edinburgh Post Natal Depression Scale; MAAS, Mindful Attention Awareness Scale; W-DEQ, Wijma Delivery Expectancy Questionnaire; DASS-21, Depression Anxiety Stress Scales.

a Significance level of one-tailed $t$-tests. $P<.05$.

b $P<.006$; this is the Bonferroni-corrected individual alpha level required to maintain experimentwise alpha at $P<.05$. 
Table 3. Significance of Changes in Outcome Measures Between Pre-MBCE Intervention and Post Birth Follow-Up for Pregnant Women $\left(\mathrm{N}=16^{\mathrm{a}}\right)$

\begin{tabular}{|c|c|c|c|}
\hline Variable (Measure) & Pre-MBCE Meana (SD) $^{a}$ & $\begin{array}{r}\text { Post Birth Follow-up } \\
\text { Mean (SD) }\end{array}$ & $P$ value $^{b}$ \\
\hline Mindfulness (MAAS) & $4.03(.84)$ & $4.63(.71)$ & $.020^{c}$ \\
\hline Fear of Birth (W-DEQ) & $64.75(13.47)$ & $41.63(22.44)$ & $.043^{c}$ \\
\hline Depression (DASS-21) & $6.00(5.51)$ & $3.09(4.76)$ & .104 \\
\hline Anxiety (DASS-21) & $9.50(7.75)$ & $3.00(4.73)$ & $<.001^{\mathrm{d}}$ \\
\hline Stress (DASS-21) & $10.91(5.00)$ & $7.82(6.66)$ & $.036^{c}$ \\
\hline
\end{tabular}

Abbreviations: MBCE, Mindfulness-Based Childbirth Education; CBSEI, Childbirth SelfEfficacy Inventory; EPNDS, Edinburgh Post Natal Depression Scale; MAAS, Mindful Attention Awareness Scale; W-DEQ, Wijma Delivery Expectancy Questionnaire; DASS-21, Depression Anxiety Stress Scales.

a Sample size reported here for follow-up varies from sample size reported in Table 2 for post-test. This is due to missing data at post-test.

b Pre-MBCE means vary between Table 1 and Table 2 due to different degrees of freedom stemming from different levels of missing data at these time points.

c Significance level of one-tailed $t$-tests. $P<.05$.

${ }^{\mathrm{d}} P<.01$; this is the Bonferroni-corrected individual alpha level required to maintain experimentwise alpha at $P<.05$. 
Table 4. Effect Sizes and Post-Hoc Power Analyses for Pre-Post MBCE Changes in Outcome Measures $(\mathrm{N}=12)$

\begin{tabular}{|c|c|c|c|c|}
\hline Variable & $\begin{array}{c}\text { Cohen's } \\
\text { d }\end{array}$ & $\begin{array}{l}\text { Effect } \\
\text { Size }^{\mathrm{a}}\end{array}$ & $\begin{array}{l}\text { Post-Hoc } \\
\text { Power }\end{array}$ & $\begin{array}{c}\text { Required } \\
\text { sample }^{b}\end{array}$ \\
\hline Self Efficacy (CBSEI) & -1.91 & Large & 1.00 & 4 \\
\hline $\begin{array}{l}\text { Outcome Expectations } \\
\text { (CBSEI) }\end{array}$ & -.75 & Medium & .79 & 13 \\
\hline $\begin{array}{l}\text { Postnatal Depression } \\
\text { (EPNDS) }\end{array}$ & .06 & Small & .07 & 1719 \\
\hline Mindfulness (MAAS) & -.52 & Medium & .51 & 25 \\
\hline Fear of Birth (W-DEQ) & 1.71 & Large & 1.00 & 4 \\
\hline Depression (DASS) & .47 & Medium & .46 & 30 \\
\hline Anxiety (DASS) & .48 & Medium & .47 & 28 \\
\hline Stress (DASS) & -.20 & Small & .16 & 161 \\
\hline
\end{tabular}

Abbreviations: MBCE, Mindfulness-Based Childbirth Education; CBSEI, Childbirth SelfEfficacy Inventory; EPNDS, Edinburgh Post Natal Depression Scale; MAAS, Mindful Attention Awareness Scale; W-DEQ, Wijma Delivery Expectancy Questionnaire; DASS-21, Depression Anxiety Stress Scales.

a Effect sizes categorized according to Cohen's ${ }^{20}$ criteria.

b Sample required to maintain statistical power at .8 . 


\section{REFERENCES}

1. Hildingsson, I. Mental training during pregnancy. Feelings and experiences during pregnancy and birth and parental stress 1 year after birth - a pilot study. Sexual and Reproductive Healthcare. 2012;3(1):31-6.

2. Huizink AC. Robles de Medina PG. Mulder E. Visser G. Buitelaar J. Stress during pregnancy is associated with developmental outcome in infancy. Journal of Child Psychology and Psychiatry. 2003;44(6):810-818.

3. Talge NM. Neal C. Glover V. Antenatal maternal stress and long-term effects on child neurodevelopment: how and why? Journal of Child Psychology and Psychiatry. 2007;48(34):245-61.

4. Baer RA. Mindfulness training as a clinical intervention: A conceptual and empirical review. Clinical Psychology: Science and Practice. 2003;10(2):125-143.

5. Duncan LG. Bardacke. Mindfulness-Based Childbirth Education and Parenting Education: Promoting Family Mindfulness During the Perinatal Period. Journal of Child and Family Studies. 2009;19(2): 190-202.

6. Wallerstein, N. Powerlessness, Empowerment, and Health: Implications for Health Promotion Programs. American Journal of Health Promotion. 1992; 6(3): 197-205.

7. Callister LC. Cultural Influences on Pain Perceptions and Behaviours. Home Health Care Management and Practice. 2003;15(3): 207-211.

8. Soet JE. Brack GA. Dilorio C. Prevalence and predictors of women's experience of psychological trauma during childbirth. Birth. 2003;30(1):36-46.

9. Nierop A. Wirtz PH. Bratsikas A. Zimmerman R. Ehlert U. Stress-buffering effects of psychosocial resources on physiological and psychological stress response in pregnant women. Biological Psychology. 2008;78(3):261-268.

10. Savage JS. The Lived Experience of Knowing in Childbirth. The Journal of Perinatal Education. 2006;15(3):10-24.

11. Fabian HM, Radestad, IJ. Waldenstrom, U. Childbirth and parenthood education classes in Sweden. Women's opinion and possible outcomes. Acta Obstetricia et Gynecologica Scandinavica. 2005 84(5):436-43.

12. Transition to Parenthood Website http://www.transitiontoparenthood.com/ttp/foreducators/handoutshome.htm. Accessed Dec 
$1,2009$.

13. Brown KW. Ryan RM. The Benefits of Being Present: Mindfulness and Its Role in Psychological Well-Being. Journal of Personality and Social Psychology 2003;84(4):822-848.

14. Lovibond, S.H. \& Lovibond, P.F. Manual for the Depression Anxiety Stress Scales. (2 ${ }^{\text {nd }}$. Ed.) Sydney: Psychology Foundation, 1995.

15. Cox JL. Holden JM. Sagovsky R. Detection of postnatal depression. Development of the 10item Edinburgh Postnatal Depression Scale. The British Journal of Psychiatry. 1987; Jun(150):782-6.

16. Lowe NK. Maternal confidence for labor: Development of the Childbirth Self-Efficacy Inventory. Research in Nursing \& Health. 1993;16(2):141-149.

17. Wijma K. Wijma B. Zar M. Psychometric aspects of the W-DEQ; a new questionnaire for the measurement of fear of childbirth. Journal of Psychosomatic Obstetrics and Gynaecology. 1998;19(2):84-97.

18. Morris SB. De Shon RP. Combining effect size estimates in meta-analysis with repeated measures and independent-groups designs. Psychological Methods. 2002;7(1):105-125.

19. Fisher, C. Hauck, Y., Bayes, S. Byrne, J. Participant Experiences of Mindfulness-Based Childbirth Education: A Qualitative Study. BMC Pregnancy and Childbirth. 2012; 12:126

20. Cohen J. Statistical Power Analysis for the Behavioral Sciences. 2nd ed. Hillsdale, NJ: Lawrence Erlbaum Associates; 1988. 